\title{
Gene evolution and gene expression after whole genome duplication in fish: the PhyloFish database
}

\author{
Jeremy Pasquier ${ }^{1}$, Cédric Cabau², Thaovi Nguyen ${ }^{1}$, Elodie Jouanno ${ }^{1}$, Dany Severac ${ }^{4}$, Ingo Braasch ${ }^{6,7}$, Laurent Journot $^{4}$, \\ Pierre Pontarotti ${ }^{5}$, Christophe Klopp ${ }^{3}$, John H. Postlethwait ${ }^{6}$, Yann Guiguen ${ }^{1+}$ and Julien Bobe ${ }^{1 *+}$
}

\begin{abstract}
With more than 30,000 species, ray-finned fish represent approximately half of vertebrates. The evolution of ray-finned fish was impacted by several whole genome duplication (WGD) events including a teleost-specific WGD event (TGD) that occurred at the root of the teleost lineage about 350 million years ago (Mya) and more recent WGD events in salmonids, carps, suckers and others. In plants and animals, WGD events are associated with adaptive radiations and evolutionary innovations. WGD-spurred innovation may be especially relevant in the case of teleost fish, which colonized a wide diversity of habitats on earth, including many extreme environments. Fish biodiversity, the use of fish models for human medicine and ecological studies, and the importance of fish in human nutrition, fuel an important need for the characterization of gene expression repertoires and corresponding evolutionary histories of ray-finned fish genes. To this aim, we performed transcriptome analyses and developed the PhyloFish database to provide (i) de novo assembled gene repertoires in 23 different ray-finned fish species including two holosteans (i.e. a group that diverged from teleosts before TGD) and 21 teleosts (including six salmonids), and (ii) gene expression levels in ten different tissues and organs (and embryos for many) in the same species. This resource was generated using a common deep RNA sequencing protocol to obtain the most exhaustive gene repertoire possible in each species that allows between-species comparisons to study the evolution of gene expression in different lineages. The PhyloFish database described here can be accessed and searched using RNAbrowse, a simple and efficient solution to give access to RNA-seq de novo assembled transcripts.
\end{abstract}

Keywords: Gene duplication, Teleosts, Holostean, Gene expression, Gar, Salmonids, Assembly, Stra8, Mcam

\section{Background}

Ray-finned fish occupy a wide diversity of aquatic habitats. More than 30,000 ray-finned fish (Actinopterygii) species have been reported that account for approximately half of vertebrates on earth [1]. A vast majority of ray-finned fish are teleosts with only 50 non-teleost species reported. Ray-finned fish evolution spanned more than 400 million years [2-4]. In addition to the two rounds of whole genome duplications that occurred at the root of the vertebrate lineage (VGD1 and VGD2)

\footnotetext{
* Correspondence: julien.bobe@rennes.inra.fr

${ }^{\dagger}$ Equal contributors

'INRA, Laboratoire de Physiologie et Génomique des poissons, Campus de Beaulieu, F-35042 Rennes cedex, France

Full list of author information is available at the end of the article
}

[5], teleost fish experienced a third round of WGD [6-8]. This teleost-specific round of WGD (TGD) occurred 320-350 million years ago (Mya), after the divergence between the holostean lineage, which includes Semionotiformes (gars) and Amiiformes (bowfin), and the lineage leading to teleost $[9,10]$. Additional WGD events have also been described in the teleost lineage [11, 12], including the salmonid-specific WGD (SaGD) that occurred about 100 Mya [13, 14].

After duplication, the most likely fate of duplicated genes is the loss of one of the duplicates through nonfunctionalization (also known as pseudogenization) that occurs by accumulation of deleterious mutations [1517]. While common after WGD, gene loss could however play a key role in speciation [18], through a process 
known as divergent resolution [19]. In addition, duplicated genes may also be retained in two copies and either specialize by the partitioning of ancestral gene functions (i.e. subfunctionalization) or by the acquisition of a novel function (i.e. neofunctionalization) [20]. In rainbow trout (Oncorhynchus mykiss), 100 million years after WGD (i.e. SaGD), 48 \% of the ancestral genes have been retained in duplicates, while $52 \%$ have resorted to singletons. Among duplicated gene pairs originating from WGD, which are also called ohnologs [21], differences are observed in the expression patterns and levels of the two copies, as shown in rainbow trout [13].

Analysis of gene expression in teleosts is therefore made interesting by the huge diversity of species $(>30,000)$, lineage-specific gene losses, differential suband neofunctionalization, and additional rounds of WGD found in this group. In addition, high quality genomic resources (i.e. fully assembled genome) remain scarce, despite a recent and substantial increase in the number of sequenced genomes publicly available. Existing fish genome resources however still lack many important nodes in teleost diversity and evolution and, for instance, more than $80 \%$ of species with sequenced genomes lie within the Euteleostei lineage, leaving out many basally diverging lineages. In line with that lack of an evolutionary based dataset of teleost genomes, our knowledge of expressed gene repertoires remains also extremely limited and skewed towards specific branches within the teleost tree of life. Significant resources exist in some lineages (e.g. percomorphs) while they are scarce in other lineages (e.g. osteoglossomorphs). The lack of data generated using similar (or at least comparable) methodologies across several species that make comparative analysis possible is a hurdle for understanding.

For reasons listed above, it is currently difficult to compare gene expression among teleost species due to (i) the lack of an exhaustive gene repertoire in all but a few species and (ii) the lack of expression data collected using comparable methodologies across the same tissues and stages in different species. The PhyloFish database was designed to address both questions and provides a comprehensive gene repertoire from de novo assembled RNA-seq data in a large number of species chosen to entirely span the ray-finned fish tree of life with special attention for TGD and SaGD WGD events. The PhyloFish database also provides consistent gene expression data in the same tissues and organs in the different species to allow between-species comparisons. The PhyloFish database is therefore a unique and essential resource to study the evolution of gene expression in the different ray-finned fish lineages that will be extremely useful in many biological fields such as 'evo-devo', ecology, toxicology, aquaculture, and physiology.

\section{Construction and content}

\section{Species selection and tissue collection}

Fish used in this study were reared and handled in strict accordance with French and European policies and guidelines of the INRA LPGP Institutional Animal Care and Use Committee (\# 25 M10), which approved this study. Species included in the PhyloFish database (Fig. 1, Table 1) were chosen not only for their evolutionary position in the tree of life $[2,22]$ but also, when possible, for their ecological and economical relevance. A total of 23 species were included in the database to span two different whole genome duplication events found in teleost fish. Different species were therefore selected before the TGD (Holosteans, $N=2$ species), after the TGD and before the SaGD (TGD teleosts, $N=15$ species), and after SaGD (SaGD teleost, $N=6$ species). Bowfin (Amia calva) and spotted gar (Lepisosteus oculatus) were selected among holosteans. Because the holostean lineage diverged from teleosts before the teleost-specific third round of whole genome (TGD) duplication, they provide useful information on the gene repertoire before the TGD and serve as an outgroup to evaluate gene evolution after the TGD. To provide a global view of gene expression patterns in TGD teleosts, 15 species were selected among the following groups: Anguilliformes, Osteoglossiformes, Clupeiformes, Cyprinformes, Siluriformes, Gymnotyformes, Characiformes, Esociformes, Osmeriformes, Gadiformes, Beloniformes, and Perciformes. While a single species was selected in most groups, three species (butterfly fish, Arowana, and elephantnose fish) were selected in the Osteoglossiformes because they diverged shortly after TGD and have few available transcriptomic resources. In addition, two species (Pike (Esox lucius) and Eastern mudminnow (Umbra pygmaea)) were selected among Esociformes as this group serves as the most closely related outgroup to study evolution after the SaGD. After SaGD, six species were selected among Salmoniformes, providing a unique opportunity to explore the evolution of gene expression and function after a comparatively recent animal genome duplication event.

For each species included in the database we constructed separate libraries from the following tissues or organs to allow analysis of tissue specific expression patterns: brain, liver, gills, heart, muscle, liver, kidney, bones, intestine, ovary, and testis. The gill library for blackghost knifefish (Apteronotus albifrons) is lacking due to limiting RNA quality libraries for embryos or larvae were made for gar (Lepisosteus oculatus), European eel (Anguilla anguilla), Aliss shad (Alosa alosa), zebrafish (Danio rerio), panga (Pangasius hypophthalmus), Northern pike (Esox lucius), grayling (Thymallus thymallus), Atlantic cod (Gadus morhua), medaka (Orysias latipes), Eurasian perch (Perca fluviatilis), brook trout 


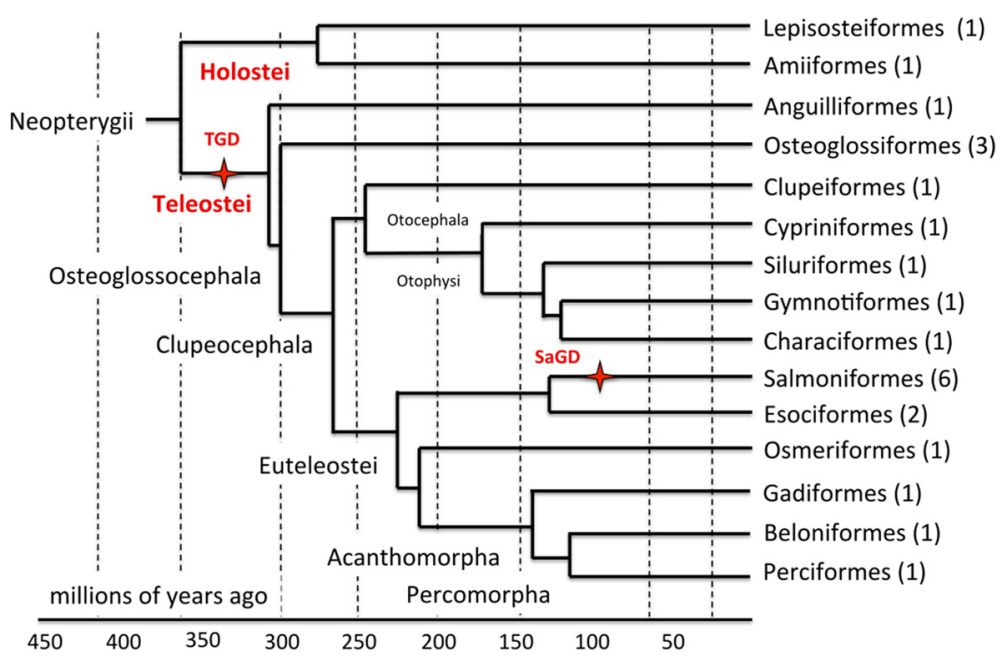

Fig. 1 Phylogenetic tree of the PhyloFish species. Cladogram showing phylogenetic relationships among ray-finned fish analyzed in the present study. Tree topology was adapted from [2]. For each phylogenetic group, the number of species in the PhyloFish set is indicated between brackets. The teleost specific (TGD) and salmonid-specific (SaGD) whole genome duplication events are indicated in red

Table 1 Species present in the PhyloFish database

\begin{tabular}{|c|c|c|c|c|}
\hline Name & Species & Phylogenetic group & $\mathrm{Nb}$ of contigs & WGD \\
\hline Bowfin & Amia calva & Amiiformes & 35064 & VGD2 \\
\hline Spotted gar & Lepisosteus oculatus & Lepisosteiformes & 41396 & VGD2 \\
\hline European eel & Anguilla anguilla & Anguilliformes & 60263 & TGD \\
\hline Butterfly fish & Pantodon buchlolzi & Osteoglossiformes & 44577 & TGD \\
\hline Arowana & Osteoglossum bicirrhosum & Osteoglossiformes & 55739 & TGD \\
\hline Elephantnose fish & Ghnathonemus petersi & Osteoglossiformes & 53423 & TGD \\
\hline Aliss shad & Alosa alosa & Clupeiformes & 53363 & TGD \\
\hline Zebrafish & Danio rerio & Cypriniformes & 48158 & TGD \\
\hline Panga & Pangasius hypophthalmus & Siluriformes & 43013 & TGD \\
\hline Black ghost knifefish & Apteronotus albifrons & Gymnotiformes & 45356 & TGD \\
\hline Mexican tetra (cave) & Astyanax mexicanus & Chraraciformes & 47729 & TGD \\
\hline Mexican tetra (surface) & Astyanax mexicanus & Characiformes & 46670 & TGD \\
\hline Northern pike & Esox lucius & Esociformes & 48567 & TGD \\
\hline Eastern mudminnow & Umbra pygmae & Esociformes & 46381 & TGD \\
\hline Grayling & Thymallus thymallus & Salmoniformes & 67157 & SaGD \\
\hline European whitefish & Coregonus lavaretus & Salmoniformes & 74701 & $\mathrm{SaGD}$ \\
\hline American whitefish & Coregonus clupeaformis & Salmoniformes & 66996 & SaGD \\
\hline Brown trout & Salmo trutta & Salmoniformes & 75338 & $\mathrm{SaGD}$ \\
\hline Rainbow trout & Oncorhynchus mykiss & Salmoniformes & 78415 & SaGD \\
\hline Brook trout & Salvelinus fontinalis & Salmoniformes & 69441 & SaGD \\
\hline Sweetfish & Pecoglossus altivelis & Osmeriformes & 47484 & TGD \\
\hline Atlantic cod & Gadus morhua & Gadiformes & 50564 & TGD \\
\hline Medaka & Orysias latipes & Beloniformes & 42186 & TGD \\
\hline European perch & Perca fluviatilis & Perciformes & 49204 & TGD \\
\hline
\end{tabular}

For each species, the common name (according to fishbase.org), the species name, phylogenetic group, the number of de novo assembled contigs generated, and position related to successive whole genome duplication (WGD) are shown. VGD2 (vertebrate $2^{\text {nd }}$ round of WGD), TGD (teleost-specific WGD), SaGD (salmonid specific WGD) 
(Salvelinus fontinalis), Mexican tetra (Astyanax mexicanus, both cave and surface populations), and European whitefish (Coregonus lavaretus). For all species, tissues were sampled from the same female individual and testis from a male individual, when possible. For rainbow trout, existing RNA-seq data previously used in the rainbow trout genome sequencing project were used [13]. In this study, tissues had been sampled from a gynogenetic female and the testis is missing. In some species and depending on the tissues, RNA samples from different individuals were pooled to obtain sufficient amounts of RNA for sequencing. All corresponding information is available in the biosample and bioproject files deposited in SRA under the PhyloFish umbrella project.

\section{RNA-seq}

Sequencing libraries were prepared using a TruSeq RNA sample preparation kit, according to manufacturer instructions (Illumina, San Diego, CA). Poly-A-containing mRNA was isolated from total RNA using poly-T oligoattached magnetic beads, and chemically fragmented. First-strand cDNA was generated using SuperScript II reverse transcriptase and random primers. Following the second strand cDNA synthesis and adaptor ligation, cDNA fragments were amplified by PCR. Products were loaded onto an Illumina HiSeq2000 instrument and subjected to multiplexed paired-end $(2 \times 100 \mathrm{bp})$ sequencing. The processing of fluorescent images into sequences, base-calling and quality value calculations were performed using the Illumina data processing pipeline.

\section{de novo transcriptome assembly}

For each library, raw sequence data in fastq format were quality checked, stored in the ng6 database [http:// www.biomedcentral.com/1471-2164/13/462], and filtered to remove unknown nucleotides. The longest subsequences without Ns exceeding half of the total read length were retained. Velvet and Oases performed transcriptome de novo assembly [23]. We first constructed nine independent assemblies for each library using different $\mathrm{k}$-mers ( $\mathrm{k}$-mers for velveth: 25,31,37,43,49,55,61,65,69; parameters for velvetg: read_trkg yes -min_contig_lgth 100 -cov_cutoff 4; parameters for oases: -cov_cutoff 4). Raw transcripts.fa files were filtered to retain only one transcript per locus based on the highest fold coverage using a Python script developed by a bioinformatic team at the Brown University (https://sites.google.com/a/brown.edu/ bioinformatics-in-biomed/velvet-and-oases-transcriptome). Antisense chimeras accidentally produced during the assembly step were removed using a homemade script. Independent assemblies were pooled and duplicate/similar transcripts built by close k-mers were removed by a cd-hitest [24] step (parameters: $-\mathrm{M} 0-\mathrm{d} 0-\mathrm{c} 0.98$ ) and merged by a TGICL [25] step (parameters: -l 60 -p 96 -s 100000). After this assembly process, all input reads were mapped back to the set of transcripts using BWA [26] and the size of the longest open reading frames (ORFs) for each transcript was computed using the getorf EMBOSS tool [27]. Finally, transcripts were filtered using mapping rate and ORF length criteria. Transcripts with ORFs shorter than $200 \mathrm{nt}$ and with fewer than two mapped reads per million of overall mapped reads were discarded. The above procedure was carried out independently for each tissuespecific library.

For each species, the library-specific assembly was followed by a meta-assembly step. The purpose of this step was to limit redundancy (i.e. identical transcript originating from different tissue libraries) in the final species-specific assembly. For each species, de novo assembled transcripts originating from the different tissuespecific libraries were pooled. The longest ORF of each transcript was extracted and ORFs were clustered using cd-hit (parameters: - M 0 -d 0 -c 0.90 -g 1). From each cd-hit cluster, the transcript with the longest ORF or the longest transcript (if more than one transcript had an ORF of the maximum size) was selected in order to increase the probability of retaining contigs with fulllength coding sequence. Input reads from all conditions were mapped back to selected transcripts using BWA. Again, transcripts were filtered based on the re-mapping rate. Transcripts with less than one mapped read per million of overall mapped reads were discarded. Finally, it should be stressed that the longest ORF was not used for annotation, because annotation was performed for each retained transcript using a BlastX procedure against existing public databases.

\section{Transcriptome coverage and quality control}

The de novo assembly procedure was trained and optimized using zebrafish, for which a genome-based high quality transcriptome is available. Our de novo assembly procedure yielded 48,158 contigs in zebrafish. This number is consistent with the 25,642 coding gene and 57,369 gene transcripts predicted from the latest Ensembl genome assembly (GRCz10, 2014). The number of PhyloFish contigs for zebrafish is lower that the total number of Ensembl zebrafish transcripts. This difference can be explained, at least in part, by the biological material used here (10 tissues, each being sampled at a single biological stage) that does not cover all possible biological conditions. The number of transcript contigs scaled with the number of WGD events, from holeosteans (two) to most teleosts (three) to salmonids (four) (Table 1). While the lowest number of contigs $(<41,500, N=2)$ was found in holosteans, it ranged between 42,200 and 60,200 in TGD teleosts $(N=15)$ and between 67,000 and 78,400 in SaGD species $(N=6)$. These figures track with the 
number of genes resulting from the different WGD events found in the analyzed species. In rainbow trout, a SaGD species, it has been shown that $48 \%$ of the duplicated genes originating from SaGD were retained in two copies [13]. The mean number of contigs in the 15 TGD but non-SaGD species present in the PhyloFish database was 48,900 , while it was 72,000 for the six SaGD salmonid species, on average. We have therefore generated $47 \%$ more contigs in SaGD species in comparison to TGD species, in agreement with the percent of duplicated gene retention after WGD in salmonids (i.e. $48 \%$ ). It should also be noted that the number of contigs was strikingly similar in the two populations of Mexican tetra that diverged recently and are therefore likely to exhibit a similar number of genes and transcripts (46,670 and 47,729 contigs were generated in surface and cave populations, respectively). Finally, when training the assembly using the zebrafish genome, we calculated that more than $75 \%$ of zebrafish contigs aligned to the zebrafish protein repertoire using BLAT with $>80 \%$ identity and $>80 \%$ coverage of the overall protein length, further validating assembly methodologies.

Together, these results indicate that the number of contigs in each species is consistent with the number of existing genes and transcripts, and that transcriptome coverage is also substantial in terms of both number of proteins and overall protein coverage despite using just 10 tissues and only one developmental timepoint.

For all species, contigs were aligned using blast against the refseq_protein and swissprot protein databases (blastx -e 1e-5 -F T -v 20 -b 20) as well as several nucleic acid databases, including Unigene Danio rerio version 126, Oryzias latipes 130 and Ensembl 71 transcripts of Danio rerio, Oryzias latipes, Takifugu rubripes and Tetraodon nigroviridis and RefSeq_RNAs (June 2013). The GO (gene ontology) annotations of aligned proteins were retrieved and stored in the database.

\section{Utility and discussion}

\section{Database features}

The PhyloFish database is made available (http://phylofish. sigenae.org/index.html) through the internet using RNAbrowse, which provides a simple and efficient access to RNA-Seq de novo assembled transcripts [28]. RNAbrowse offers many features that will help users analyze and extract biologically meaningful information from the PhyloFish data. The PhyloFish web browser offers several different possible modes of analysis. For each species, which can be selected in the front page by a simple click on the species name, an overview is provided that includes a set of graphics showing general statistics, containing for example the contig length histogram. The browser also includes detailed information about the different sequenced libraries and provides access to tools such as Venn diagrams and digital differential display. A blast query form is available to align a known sequence on all contigs. The query must be provided using a fasta or multi fasta format. The search can also be done using a name or description through the biomart form. Users can then add retrieved contigs to the favorite table. For each contig, the sequence can be extracted to perform a multiple alignment to check if different splice forms have been assembled. All possible open reading frames can be visualized and annotations can be graphically displayed using jbrowse [29]. It is also possible to graphically visualize expression levels along the contigs in the different libraries. Expression data in the various libraries can be exported to generate expression profiles for the different tissues/organs. To our knowledge, the PhyloFish database is the only database that allows (i) the identification of contigs in such a large diversity of fish species, including many species with no or limited transcriptomic resources, and (ii) the generation of tissue expression patterns from 23 different species (including two holosteans) in which the same tissues were sampled by consistent methodologies and for which the RNA-seq procedure is similar (i.e. with the same chemistry, the same type of library, and the same sequencing depth), all features that promote normalized comparisons across tissues and taxa.

\section{Case study}

To illustrate the utility of the PhyloFish database to solve problems of gene evolutionary history, we used it to decipher the evolutionary history of stra8 (Stimulated by retinoic acid gene 8 ), and subsequently characterize its expression in holosteans and teleosts. The stra 8 gene encodes a retinoic acid-responsive protein that is involved in the regulation of meiotic initiation during spermatogenesis and oogenesis [30]. This gene was first hypothesized to be lost either in the ray-finned fish lineage or in the teleost lineage [31]. This assumption was mainly based on its absence from the zebrafish genome and other teleost reference genomes available at that time (i.e. stickleback, Tetraodon, fugu, medaka). The loss of stra 8 in teleosts was however recently challenged by the discovery of an apparent stra8 ortholog (AGM53488.1) in Southern catfish (Silurus meridionalis) [32]. We revisited stra 8 gene evolution using the PhyloFish database as a main resource. Using the Stra8 protein sequence from Southern catfish [32], we queried PhyloFish databases and retrieved fourteen sequences with a significant Stra8 hit in thirteen teleosts and one holostean species (species in bold type in Fig. 2a). These fourteen sequences were used in a phylogenetic analysis combined with the Southern catfish Stra8 protein sequence used as bait in our analysis and three additional teleost Stra8 sequences available in public databases (Esox lucius, Astyanax mexicanus, and Anguilla japonica). Phylogenetic analysis revealed that all these PhyloFish sequences 

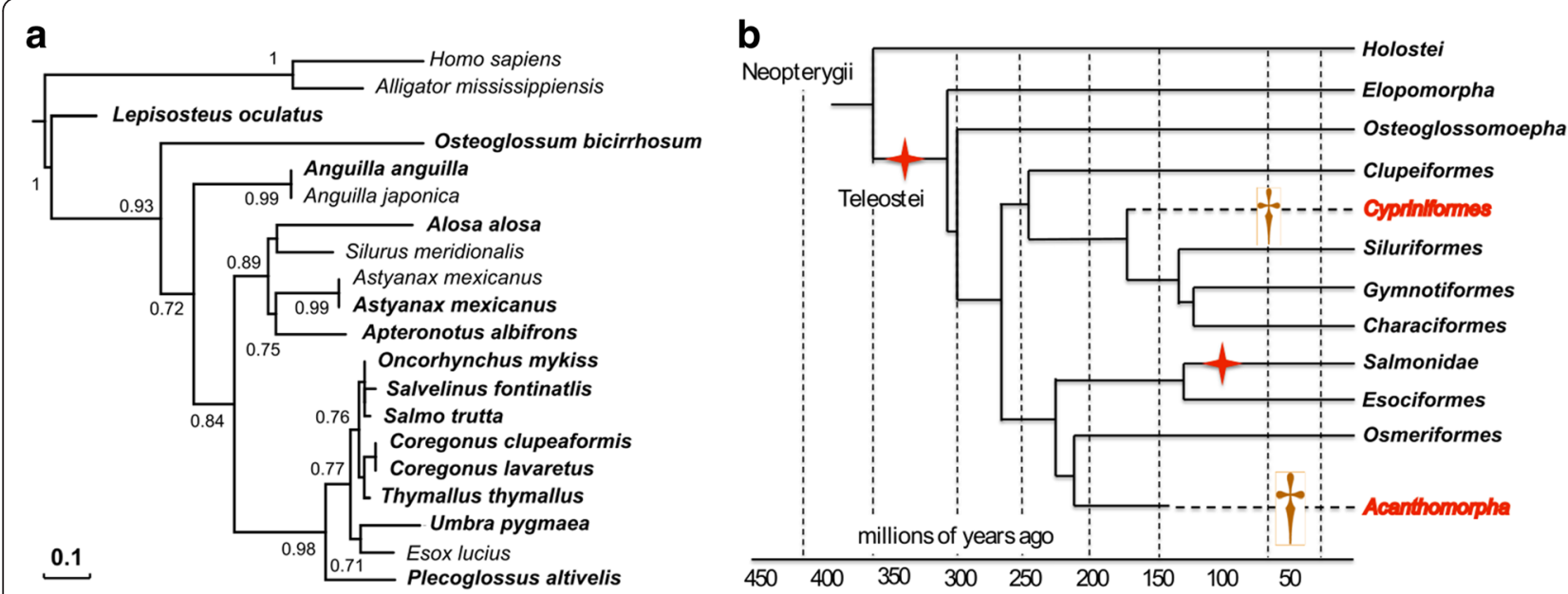

Fig. 2 Stra8 proposed gene evolution in teleosts following the TGD WGD. Maximum-likelihood phylogeny of Stra8 (a) was performed using the PhyML software [38] implemented in the Phylogeny.fr web platform [39] using default "a la carte" parameters and a bootstrapping procedure ( $N=100$ bootstraps). The resulting tree was exported and edited in Evolview [40]. Input sequences were retrieved using a tblastn search of the PhyloFish database using as bait the Southern catfish Stra8 protein (AGM53488.1). PhyloFish Stra8 coding sequences (in bold on the tree) were submitted to GenBank with the following accession numbers: Lepisosteus oculatus (KU161162), Osteoglossum bicirhosum (KU161164), Anguilla anguilla (KU161163), Alosa alosa (KU161165), Astyanax mexicanus (KU161166), Apteronotus albifrons (KU161167), Oncorhynchus mykiss (KU161168), Salvelinus fontinalis (KU161169), Coregonus lavaretus (KU161172), Coregonus clupeaformis (KU161171), Salmo trutta (KU161170), Thymallus thymallus (KU161173), Umbra pygmae (KU161174) and Plecoglossus altivelis (KU161175). This dataset was complemented with two additional teleost public sequences of Stra8 in Esox lucius (XP_012986862) and Astyanax mexicanus (XP_007229918.1) and a Stra8 sequence deduced from the Anguilla japonica genome (scaffold 6093). The tree was rooted with tetrapod sequences using the Homo sapiens STRA8 (AAP47163.1) and Alligator mississippiensis Stra8 (XP_006261218.1). b Schematic representation of the deduced evolution of stra8 based on PhyloFish sequences. This analysis suggests that stra8 was completely lost in Acanthomorpha, but also specifically and independently lost in the Cypriniformes lineage. The tree is based on [2]

are true orthologs of the tetrapods and the southern catfish stra 8 gene (Fig. 2a) and that only a single stra 8 paralog was retained after the TGD whole genome duplication. No stra 8 sequence was found in the PhyloFish database for zebrafish, cod, medaka, and European perch, thus corroborating previous reports based on zebrafish and Acanthomorpha genome analysis [31]. No stra 8 homolog was detected in public databases in any Cypriniform species (e.g., carps) even after an extensive search of GenBank nucleotide collection (nr/nt), Expressed Sequence Tags (ESTs), Transcriptome Shotgun Assembly (TSA), and NCBI genomes. This surprising finding strongly suggests that stra8 was lost in Acanthomorpha (Fig. 2b), and independently lost in the Cypriniform lineage. In addition, using the PhyloFish database, we explored the tissue expression of stra 8 genes, showing that stra 8 is mainly gonadal with a predominant expression in the testis (Fig. 3), as previously shown in the Southern catfish [32] and in mammals [33].

In addition to the stra 8 case study presented above that highlights a very specific case of evolution after duplication (i.e., loss of one copy of a duplicated pair of paralogs and lineage-specific losses of the second copy) we also investigated a more classical case of gene evolution. We thus characterized the evolutionary history of mcam (melanoma cell adhesion molecule) (also known as $c$ 1146). This gene encodes a protein with known roles in cell adhesion and in cohesion of the endothelial monolayer at intercellular junctions in vascular tissue $[34,35]$. Using a combination of sequences originating from sequences available in GenBank and from the PhyloFish database, we reconstructed the evolutionary history of the mcam gene (Fig. 4). This gene was retained as two paralogous copies after the TGD with an additional complete retention of duplicated paralogs in the salmonid lineage after the SaGD. This gene follows a complete 1 (Tetrapods and Holostei) to 2 (Teleosts) to 4 (Salmonids) duplication rule with a total retention of paralogs after two round of whole genome duplication leading to four copies in salmonids.

PhyloFish data were also used to characterize the evolution of the expression of prrx 1 and prrx 2 genes, two VGD ohnologs, in teleosts compared to the spotted Gar. We concluded that for prrx, the spotted gar genome and gar gene expression patterns mimic mammals better than teleosts do, and that there is significant diversity among teleost lineages with respect to the loss and retention of prrx TGD ohnologs [36]. Finally, the PhyloFish database was recently used by the Spotted Gar Genome Consortium to thoroughly analyze the evolution of gene expression after TGD using spotted gar, zebrafish, and medaka [37]. 

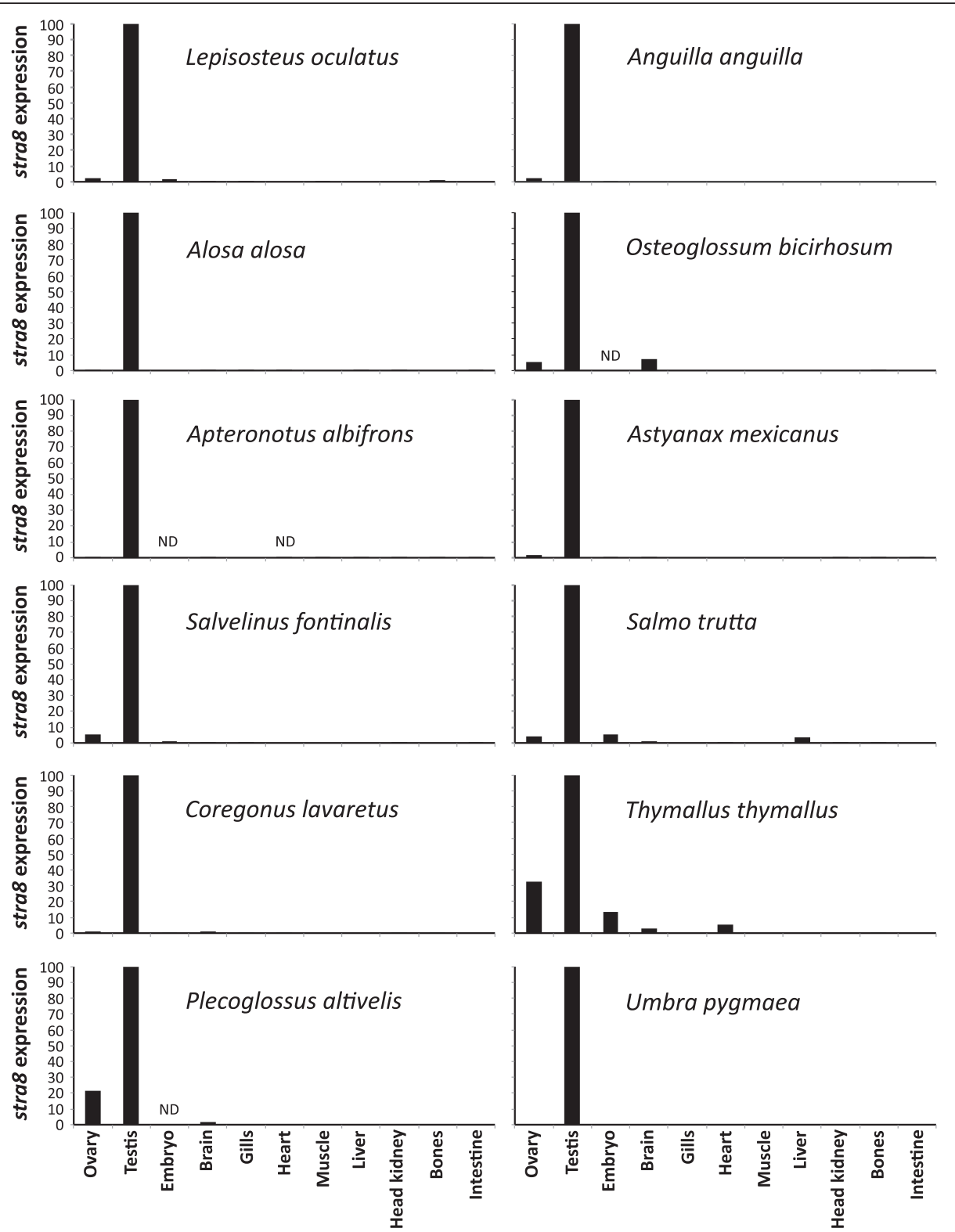

Fig. 3 Tissue expression profiles of stra8 reveal expression predominantly in testes in most PhyloFish species. Relative expression of stra8 was calculated as the percentage of the maximum rpkm (number of reads per kilobase per million reads) value per species. ND: no data (tissue not sequenced in that species)

\section{Conclusions}

The PhyloFish database is a unique resource providing comprehensive expressed gene repertoires collected and processed using the same protocol for 23 ray-finned fish species. This resource is currently the only database offering the possibility to analyze gene expression after genome duplication in teleost fish, including salmonids, in such a comprehensive and comparative way. The PhyloFish database has already proved its utility and will be of further interest in many biological fields such as 'evodevo', ecology, toxicology, aquaculture, and physiology. In the future, the PhyloFish database can be expanded to incorporate data from other fish species to broaden its scope and explore gene evolution in many different teleost lineages.

\section{Availability and requirements}

The PhyloFish database is available online at http:// phylofish.sigenae.org/index.html. All sequences described in this paper can be downloaded from that site. RNA-seq raw sequence data from the Hiseq2000 sequencer have been deposited into the NCBI SRA under accessions SRP044781 (zebrafish), SRP044782 (spotted gar), SRP044783 (bowfin), SRP044784 (medaka), SRP045098 


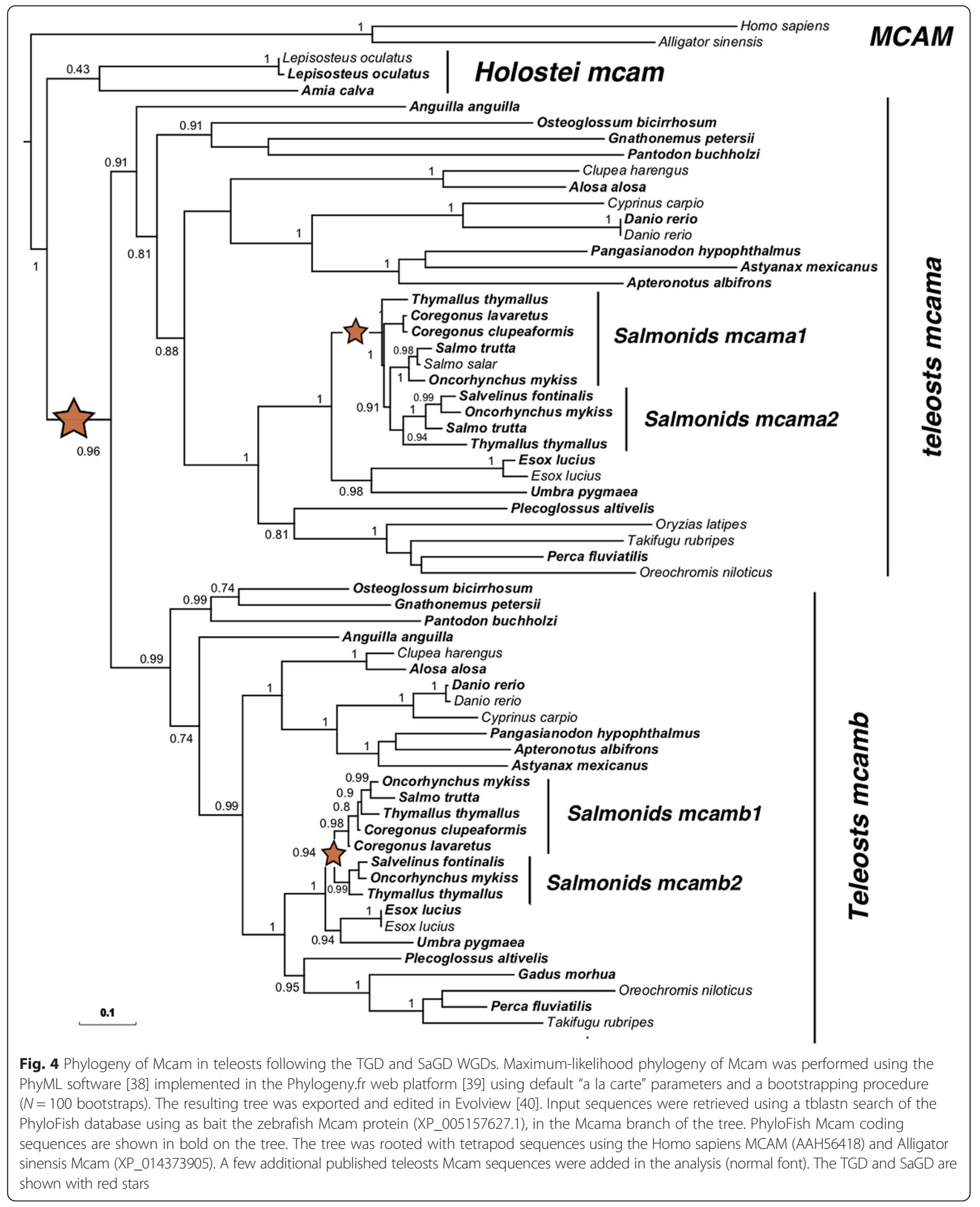

(black ghost knifefish), SRP045099 (European eel), SRP045100 (butterfly fish), SRP045101 (brown trout), SRP045102 (arowana), SRP045103 (aliss shad), SRP045138 (eastern mudminnow), SRP045139 (rainbow trout), SRP 045140 (panga), SRP045141 (northern pike), SRP045142 (grayling), SRP045143 (European whitefish), SRP045144 
(European perch), SRP045145 (elephantnose fish), SRP 045146 (sweetfish), SRP058861 (lake whitefish), SRP058862 (brook trout), SRP058863 (cave fish), SRP058865 (Atlantic cod), and SRP058863 (surface fish).

\section{Competing interests}

The authors declare that they have no competing interests.

\section{Authors' contributions}

EJ and TN participated in tissue collection, RNA extraction and histological sex phenotyping. HP and LJ constructed libraries and performed RNA-seq. CC and CK performed bioinfomatic analyses. IB, PP and JP participated in manuscript writing and data analysis. YG, JHP and JB conceived the study, participated in tissue collection, analyzed results and drafted the manuscript. All authors read and approved the final manuscript.

\section{Acknowledgements}

This work was supported by the French national research Agency (ANR-10GENM-017-PhyloFish to JB, ANR-13-BSV7-0015-Maternal Legacy to JB, and ANR-13-ISV7-0005-PhyloSex to YG) and by the National Institutes of Health (R01 OD011116 and R24 OD011199 to JHP). The authors thank the following persons for their precious help in providing fish and / or fish samples: Allyse Ferrara and Quenton Fontenot (Nicholls State University, USA) for bowfin and spotted gar, Benjamin Geoffroy and Agnes Bardonnet (UMR INRA/UPPA, St Pée-sur-Nivelle, France) for European eel, Denis Clavé (Migado, Mouleydier, France) for Aliss shad, Marc Legendre and Jean-Christophe Avarre (IRD, Montpellier, France) for Panga, Sylvie Retaux (CNRS-DECA, Gif-sur-Yvette, France) for Mexican tetra, Pascal Fontaine (Lorraine University, Nancy, France) for Northern pike and European perch, Hugo Verreycken (INBO, Brussels, Belgium) for Eastern mudminnow, Martin Gerber (Fédération de pêche du Bas-Rhin, Obenheim, France) for Grayling, Cyrille Chataigner (salmoniculture de Rives, Thonon-les-Bains, France) for European whitefish, Louis Bertnatchez (Laval University, Canada) for American whitefish and for leptocephalus European eel larval stage, Goro Yoshizaki (Tokyo University of Marine Science and Technology, Tokyo, Japan) for Sweetfish, and Hervé Migaud (Institute of Aquaculture, Stirling, Scotland) for Atlantic cod.

\section{Author details}

'INRA, Laboratoire de Physiologie et Génomique des poissons, Campus de Beaulieu, F-35042 Rennes cedex, France. ${ }^{2}$ INRA, SIGENAE, GenPhySE, F-31326 Castanet-Tolosan, France. ${ }^{3}$ INRA, SIGENAE, UR 875, MIAT INRA, Toulouse, France. ${ }^{4}$ CNRS, MGX-Montpellier GenomiX, Montpellier, France. ${ }^{5}$ Aix-Marseille Université, CNRS, Centrale Marseille, I2M, UMR7373, FR 4213 - FR, Eccorev 3098, équipe EBM, 13331 Marseille, France. ${ }^{6}$ Institute of Neuroscience, University of Oregon, Eugene 97403-1254, OR, USA. ${ }^{7}$ Department of Integrative Biology, Michigan State University, East Lansing 48824, MI, USA.

Received: 19 December 2015 Accepted: 5 May 2016

Published online: 18 May 2016

\section{References}

1. Nelson J. Fishes of the World. 2006

2. Near TJ, Eytan Rl, Dornburg A, Kuhn KL, Moore JA, Davis MP, Wainwright PC, Friedman M, Smith WL. Resolution of ray-finned fish phylogeny and timing of diversification. Proc Natl Acad Sci U S A. 2012;109:13698-703.

3. Betancur-R R, Broughton RE, Wiley EO, Carpenter K, López JA, Li C, et al. The tree of life and a new classification of bony fishes. PLoS Curr. 2013;5.

4. Broughton RE, Betancur-R R, Li C, Arratia G, Ortí G. Multi-locus phylogenetic analysis reveals the pattern and tempo of bony fish evolution. PLoS Curr. 2013;5. doi: 10.1371/currents.tol.2ca8041495ffafd0c92756e75247483e

5. Dehal P, Boore JL. Two rounds of whole genome duplication in the ancestral vertebrate. PLoS Biol. 2005;3(10):e314.

6. Amores A, Force A, Yan YL, Joly L, Amemiya C, Fritz A, Ho RK, Langeland J, Prince V, Wang YL, Westerfield M, Ekker M, Postlethwait JH. Zebrafish hox clusters and vertebrate genome evolution. Science. 1998;282(5394):1711-4

7. Van de Peer Y, Taylor JS, Meyer A. Are all fishes ancient polyploids? J Struct Funct Genomics. 2003;3(1-4):65-73.

8. Meyer A, Van de Peer Y. From 2R to 3R: evidence for a fish-specific genome duplication (FSGD). Bioessays. 2005;27(9):937-45.
9. Hoegg S, Brinkmann H, Taylor JS, Meyer A. Phylogenetic timing of the fishspecific genome duplication correlates with the diversification of teleost fish. J Mol Evol. 2004:59:190-203.

10. Amores A, Catchen J, Ferrara A, Fontenot Q, Postlethwait JH. Genome evolution and meiotic maps by massively parallel DNA sequencing: spotted gar, an outgroup for the teleost genome duplication. Genetics. 2011;188: 799-808.

11. Uyeno T, Smith GR. Tetraploid origin of the karyotype of catostomid fishes. Science. 1972:175:644-6.

12. Larhammar D, Risinger C. Molecular genetic aspects of tetraploidy in the common carp Cyprinus carpio. Mol Phylogenet Evol. 1994;3:59-68.

13. Berthelot $C$, Brunet $F$, Chalopin D, Juanchich A, Bernard M, Noël B, Bento P, Da Silva C, Labadie K, Alberti A, Aury J-M, Louis A, Dehais P, Bardou P, Montfort J, Klopp C, Cabau C, Gaspin C, Thorgaard GH, Boussaha M, Quillet E, Guyomard R, Galiana D, Bobe J, Volff J-N, Genêt C, Wincker P, Jaillon O, Roest Crollius $\mathrm{H}$, Guiguen $Y$. The rainbow trout genome provides novel insights into evolution after whole-genome duplication in vertebrates. Nat Commun. 2014;5:3657.

14. Macqueen DJ, Johnston IA. A well-constrained estimate for the timing of the salmonid whole genome duplication reveals major decoupling from species diversification. Proc Biol Sci. 2014;281:20132881.

15. Nei M, Roychoudhury AK. Probability of fixation and mean fixation time of an overdominant mutation. Genetics. 1973:74:371-80.

16. Takahata N, Maruyama T. Polymorphism and loss of duplicate gene expression: a theoretical study with application of tetraploid fish. Proc Nat Acad Sci U S A. 1979:76:4521-5

17. Watterson GA. On the time for gene silencing at duplicate Loci. Genetics. 1983:105:745-66.

18. Lynch $\mathrm{M}$, Conery JS. The evolutionary fate and consequences of duplicate genes. Science. 2000;290:1151-5.

19. Taylor JS, Van de Peer Y, Meyer A. Genome duplication, divergent resolution and speciation. Trends Genet. 2001;17:299-301.

20. Force A, Lynch M, Pickett FB, Amores A, Yan YL, Postlethwait J. Preservation of duplicate genes by complementary, degenerative mutations. Genetics. 1999;151:1531-45

21. Wolfe KH. Origin of the Yeast Whole-Genome Duplication. PLoS Biol. 2015; 13:e1002221.

22. Near TJ, Dornburg A, Eytan RI, Keck BP, Smith WL, Kuhn KL, Moore JA, Price SA, Burbrink FT, Friedman M, Wainwright PC. Phylogeny and tempo of diversification in the superradiation of spiny-rayed fishes. Proc Natl Acad Sci U S A. 2013;110:12738-43.

23. Schulz MH, Zerbino DR, Vingron M, Birney E. Oases: robust de novo RNAseq assembly across the dynamic range of expression levels. Bioinformatics. 2012:28:1086-92.

24. Li W, Godzik A. Cd-hit: a fast program for clustering and comparing large sets of protein or nucleotide sequences. Bioinformatics. 2006;22:1658-9.

25. Pertea G, Huang X, Liang F, Antonescu V, Sultana R, Karamycheva S, Lee Y, White J, Cheung F, Parvizi B, Tsai J, Quackenbush J. TIGR Gene Indices clustering tools (TGICL): a software system for fast clustering of large EST datasets. Bioinformatics. 2003;19:651-2.

26. Li H, Durbin R. Fast and accurate short read alignment with BurrowsWheeler transform. Bioinformatics. 2009;25:1754-60.

27. Rice $\mathrm{P}$, Longden I, Bleasby A. EMBOSS: the European Molecular Biology Open Software Suite. Trends Genet. 2000;16:276-7.

28. Mariette J, Noirot C, Nabihoudine I, Bardou P, Hoede C, Djari A, Cabau C, Klopp C. RNAbrowse: RNA-Seq de novo assembly results browser. PLoS One. 2014;9:e96821.

29. Westesson O, Skinner M, Holmes I. Visualizing next-generation sequencing data with JBrowse. Brief Bioinform. 2013;14:172-7.

30. Feng C-W, Bowles J, Koopman P. Control of mammalian germ cell entry into meiosis. Mol Cell Endocrinol. 2014;382:488-97.

31. Rodríguez-Marí A, Cañestro C, BreMiller RA, Catchen JM, Yan Y-L, Postlethwait JH. Retinoic acid metabolic genes, meiosis, and gonadal sex differentiation in zebrafish. PLOS One. 2013:8:e73951.

32. Dong $R$, Yang S, Jiao J, Wang T, Shi H, Zhou L, Zhang Y, Wang D. Characterization of Stra8 in Southern catfish (Silurus meridionalis): evidence for its role in meiotic initiation. BMC Mol Biol. 2013;14:11.

33. Miyamoto T, Sengoku K, Takuma N, Hasuike S, Hayashi H, Yamauchi T, Yamashita T, Ishikawa M. Isolation and expression analysis of the testisspecific gene, STRA8, stimulated by retinoic acid gene 8. J Assist Reprod Genet. 2002;19:531-5. 
34. Chan B, Sinha S, Cho D, Ramchandran R, Sukhatme VP. Critical roles of CD146 in zebrafish vascular development. Dev Dyn. 2005;232:232-44.

35. Wang Z, Yan X. CD146, a multi-functional molecule beyond adhesion. Cancer Lett. 2013;330:150-62.

36. Braasch I, Guiguen Y, Loker R, Letaw JH, Ferrara A, Bobe J, Postlethwait JH. Connectivity of vertebrate genomes: Paired-related homeobox (Prrx) genes in spotted gar, basal teleosts, and tetrapods. Comp Biochem Physiol C Toxicol Pharmacol. 2014;163:24-36.

37. Braasch I, Gehrke AR, Smith JJ, Kawasaki K, Manousaki T, Pasquier J, Amores A, Desvignes T, Batzel P, Catchen J, Berlin AM, Campbell MS, Barrell D, Martin KJ, Mulley JF, Ravi V, Lee AP, Nakamura T, Chalopin D, Fan S, Wcisel D, Cañestro C, Sydes J, Beaudry FEG, Sun Y, Hertel J, Beam MJ, Fasold M, Ishiyama $\mathrm{M}$, Johnson J, et al. The spotted gar genome illuminates vertebrate evolution and facilitates human-teleost comparisons. Nat Genet. 2016;48:427-37.

38. Guindon S, Dufayard J-F, Lefort V, Anisimova M, Hordijk W, Gascuel O. New algorithms and methods to estimate maximum-likelihood phylogenies: assessing the performance of PhyML 3.0. Syst Biol. 2010;59:307-21.

39. Dereeper A, Guignon V, Blanc G, Audic S, Buffet S, Chevenet F, Dufayard J-F, Guindon S, Lefort V, Lescot M, Claverie J-M, Gascuel O. Phylogeny.fr: robust phylogenetic analysis for the non-specialist. Nucleic Acids Res. 2008;36(Web Server issue):W465-9.

40. Zhang H, Gao S, Lercher MJ, Hu S, Chen W-H. EvolView, an online tool for visualizing, annotating and managing phylogenetic trees. Nucleic Acids Res. 2012;40(Web Server issue):W569-72.

\section{Submit your next manuscript to BioMed Central} and we will help you at every step:

- We accept pre-submission inquiries

- Our selector tool helps you to find the most relevant journal

- We provide round the clock customer support

- Convenient online submission

- Thorough peer review

- Inclusion in PubMed and all major indexing services

- Maximum visibility for your research

Submit your manuscript at www.biomedcentral.com/submit

) Biomed Central 